\title{
Anistropic hole formation in thin polymer films confined by walls
}

\author{
K. Y. Suh and Hong H. Lee ${ }^{\text {a) }}$ \\ School of Chemical Engineering, Seoul National University, Seoul, 151-742 Korea
}

(Received 10 May 2001; accepted 17 August 2001)

We report dewetting behavior of thermally annealed thin polymer strips of poly (styrene-b-butadiene-b-styrene) and polystyrene films that are laterally confined by polydimethylsiloxane walls on silicon substrate. Regularly spaced holes are initially nucleated on the polymer surface and then grow with the aid of the confining walls, resulting in the formation of distinctly observable, regularly spaced blocks of the strip. It is observed that the dependence of hole density on the film thickness severely deviates from the conventional capillary wave model. This anomaly may be related to the viscoleastic properties of the polymer film. (C) 2001 American Institute of Physics. [DOI: 10.1063/1.1409540]

\section{INTRODUCTION}

Dewetting of a liquid film on a substrate has drawn much interest in the last ten years due to fundamental interest in basic wetting phenomena and potential applications to nano-patterning technology. ${ }^{1-9}$ Much of the theoretical and experimental work has been devoted to polymer films because of the technological importance and convenient experimental time scale that polymer films offer due to their high viscosity. ${ }^{3-5,8-9}$

Although there are still debates on the exact mechanism of the dewetting process, it has been reported that the initial state of dewetting is governed by the instability driven by the dispersion forces that is known as spinodal dewetting. ${ }^{1}$ The resulting morphologies of spinodal dewetting take on two types, depending on the film thickness and molecular weight. One is nucleation of holes, ${ }^{3}$ which takes place for intermediate film thickness $(<1 \mu \mathrm{m})$ that is accelerated by the presence of small particles (i.e., dust) or imperfections on the surface. $^{2}$ The other is unstable growth of a capillary wave, ${ }^{5}$ which takes hold for very thin films of polymer (typically $<10 \mathrm{~nm}$ ) and low molecular weight $\left(<10^{4}\right)$. Sometimes a capillary wave leads to a bicontinuous shape. ${ }^{5}$

Recently, anisotropic spinodal dewetting of polymer films has been reported ${ }^{9}$ as a route to patterning surfaces on nanometer scale. Due to the isotropic nature in spinodal dewetting, the authors used chemically or mechanically treated substrates, which gives rise to the anisotropic behavior.

In this paper, we present dewetting behavior of laterally confined polymer films with an experimental method specifically devised for the purpose. We used polydimethylsiloxane (PDMS) walls as a confining medium and observed anisotropic hole formation without any chemical or mechanical treatment of the substrate. We found that the dewetting wave propagates only along the strip direction and that the dewetting results in the formation of a directly observable, regular structure.

\footnotetext{
${ }^{\text {a) }}$ Author to whom correspondence should be addressed. Electronic mail: honghlee@plaza.snu.ac.kr
}

\section{EXPERIMENTAL METHOD}

For the experimental setup, we fabricated polydimethylsiloxane (Sylgard 184, Dow Corning) mold that has a planar surface with recessed patterns by casting PDMS against a complementary relief structure prepared by photolithographic method. ${ }^{10}$ The line-and-space pattern on the mold has the lines varying in width from $800 \mathrm{~nm}$ to $3 \mu \mathrm{m}$ and a step height of $550 \mathrm{~nm}$. As shown in Fig. 1(a), the mold with the pattern is placed on the surface of a polymer layer spincoated onto a substrate and then heated above the glass transition temperature of the polymer. Capillary force then allows the polymer melt to fill up the void space of the channels formed between the mold and the polymer, thereby generating a laterally confined geometry. In other words, each of the polymer strips (lines) formed in each of the channels is confined by silicon substrate and two PDMS walls. The whole structure is then left undisturbed at the chosen heating temperature in air to prevent the separation of the mold and let the dewetting take place. After a period of time, the PDMS mold is removed after cooling to room temperature. The pattern resulting from the dewetting, as illustrated in Fig. 1(b), was examined by optical microscopy, scanning electron microscopy (SEM) and atomic force microscopy (AFM).

For the polymer, we used commercial polystyrene (PS) (molecular weight $=2.3 \times 10^{5}, T_{g}=101^{\circ} \mathrm{C}$ ) and symmetric styrene-butadiene-styrene (SBS) block copolymer (molecular weight $=3 \times 10^{5}$, styrene fraction $=0.3$, and $T_{g}$ $=-36{ }^{\circ} \mathrm{C}$ ). Silicon wafer that was used as the substrate was cleaned by ultrasonic treatment in trichloroethylene and methanol for five minutes each and dried in nitrogen. Native oxide was not removed of the surface and thus may exist on the surface. Polymer films were spin-coated onto the substrate to $42-127 \mathrm{~nm}$ thickness.

\section{RESULTS AND DISCUSSION}

If the film is thick enough such that the mold void is completely filled, no dewetting takes place. When the film is 


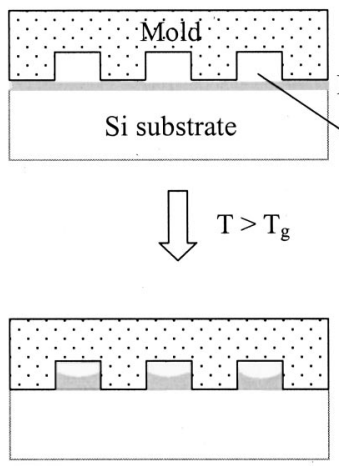

(a)
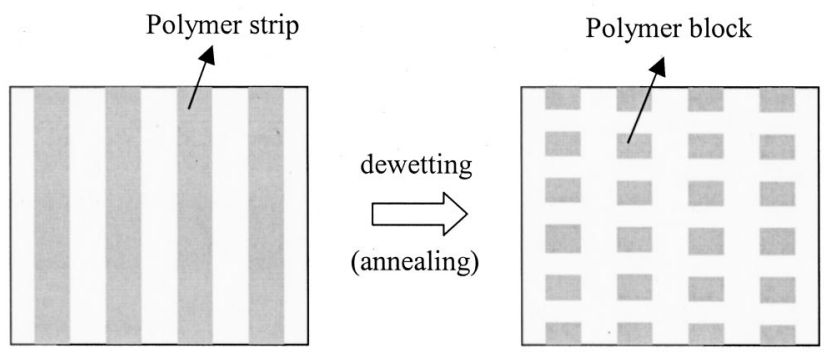

(b)

FIG. 1. (a) Schematic diagram of the experimental setup where $T_{g}$ indicates the glass transition temperature. (b) Illustration of separated block formation from originally full polymer strips.

thin such that the mold void is partially filled as in Fig. 1(a), however, the hole formation occurs above the glass transition temperature.

Figure 2 shows optical images of the typical transient morphologies of laterally confined SBS films for a $1 \mu \mathrm{m}$ line-and-space pattern that results from the dewetting. The pattern consists of six strips: five separated and one isolated lines. The samples were annealed at $120^{\circ} \mathrm{C}$ for various annealing times. Up to $19 \mathrm{~h}$, there is no observable change on the polymer surface as shown in Fig. 2(a). However, regularly spaced holes are detected after $21 \mathrm{~h}$ [Fig. 2(b)] and some holes grow across the strip [Fig. 2(c)]. Finally, all the holes propagate to the edge in the lateral direction, which leads to the regularly separated block formation [Fig. 2(d)]. The cross sectional SEM image of Fig. 2(d) is shown in Fig. 2(e), which clearly shows the periodic block formation along the strip.

In order to further examine the transient morphology, we carried out AFM measurements for a $3 \mu \mathrm{m}$ line-and-space pattern of SBS film. As shown in Fig. 3(a) by circles, regularly spaced holes are initially nucleated on the polymer surface and grow in size, contacting one of the mold walls. It has been reported that PS film is unstable on the PDMS surface. ${ }^{11}$ If we assume that SBS has similar properties as those of PS, SBS is also unstable on PDMS surface above their glass transition temperatures such that the walls facilitate the hole growth. Once the hole contacts the wall, it then grows across the strip. Propagation of the growth to the other wall leads to the formation of separated blocks of polymer
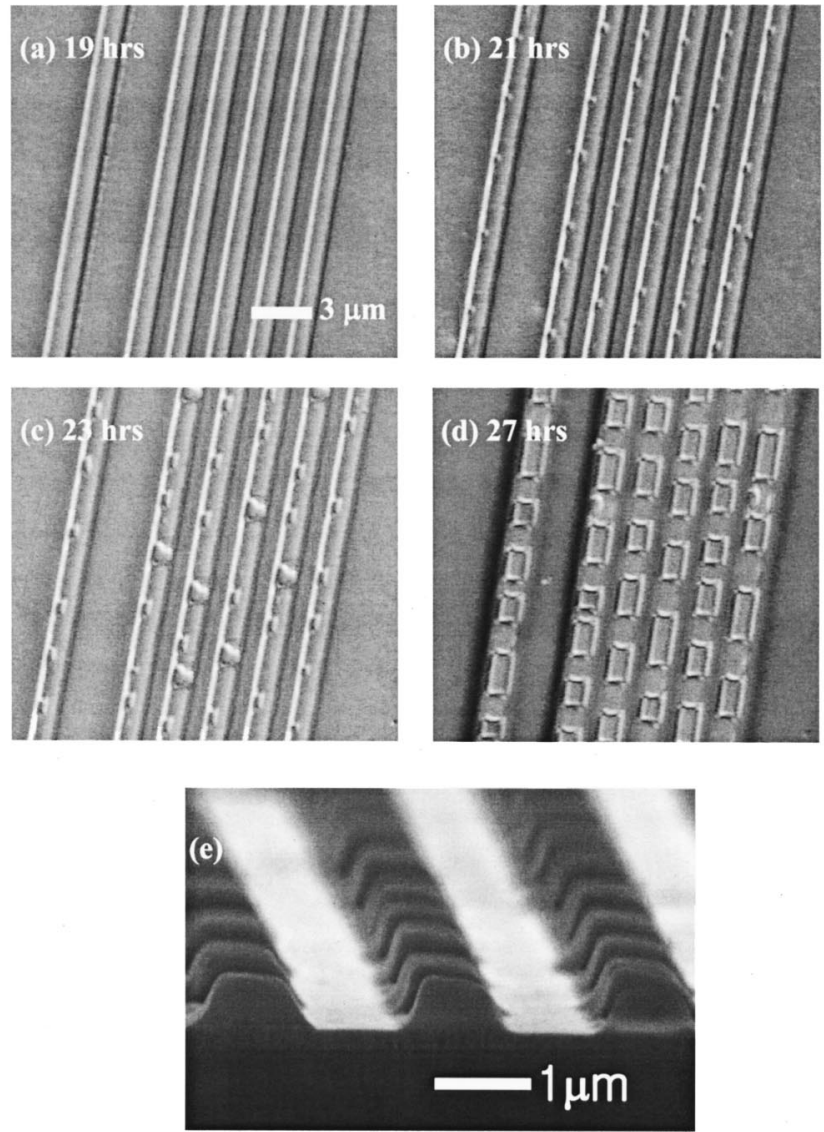

FIG. 2. Optical [(a) to (d)] and cross sectional scanning electron microscopy (SEM) (e) images of transient morphologies of SBS thin films for $1 \mu \mathrm{m}$ line-and-space pattern. The film thickness is about $67 \mathrm{~nm}$ and the samples are annealed at $120^{\circ} \mathrm{C}$ for various annealing times. (e) The cross sectional view of (d) across the strip width.

strip with a certain periodicity as can be seen in Figs. 2(d), 2(e), and 3(b). The film thickness in Fig. 3 is about $67 \mathrm{~nm}$ initially, but the film thickness of the molded polymer strip that was formed in the channel is $125 \mathrm{~nm}$ due to the filling of the void space by capillary flow. After the formation of separated blocks, the thickness increases further to $287 \mathrm{~nm}$ due to further mass transport and there was little polymer left in the region between the blocks. However, two narrow peripheral lines form, of about $25 \mathrm{~nm}$ high, that connect two adjacent blocks.

It is noted that the existence of PDMS walls is a critical factor for the formation of separated polymer blocks. Without the walls, the nucleated holes could not laterally grow across the wall direction, thereby leading to the isolated hole structures. In addition, the confining walls give rise to an anisotropic hole growth by suppressing the wave propagation in one direction. We found that the anisotropic nature is destroyed as the strip width increases. The critical value above which the anisotropy is destroyed is about $8 \mu \mathrm{m}$.

The incubation time that is needed for the onset of the instability depends on the film thickness and annealing temperature. For example, it is about $20 \mathrm{~h}$ for $67-\mathrm{nm}$-thick films and about $12 \mathrm{~h}$ for 42 -nm-thick films at $120^{\circ} \mathrm{C}$ for SBS. If the temperature increases to $150^{\circ} \mathrm{C}$, the incubation 


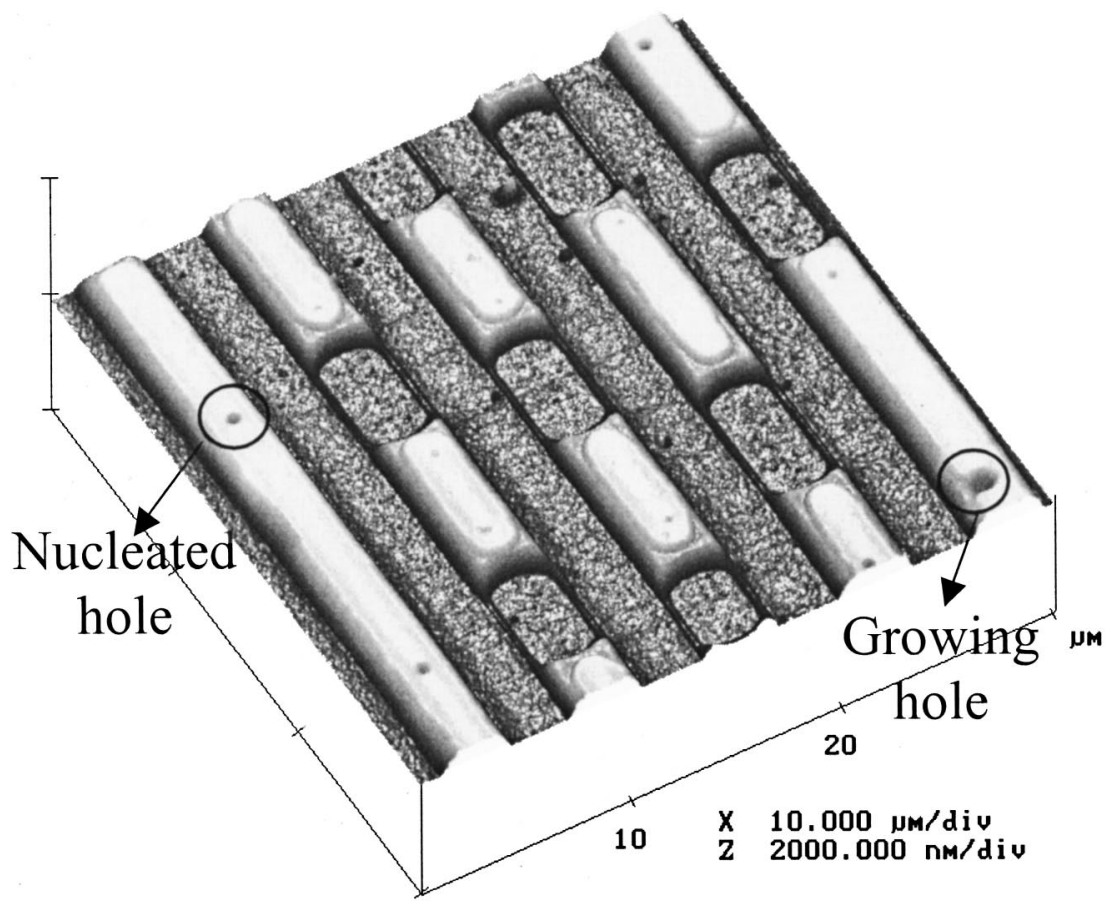

(a)

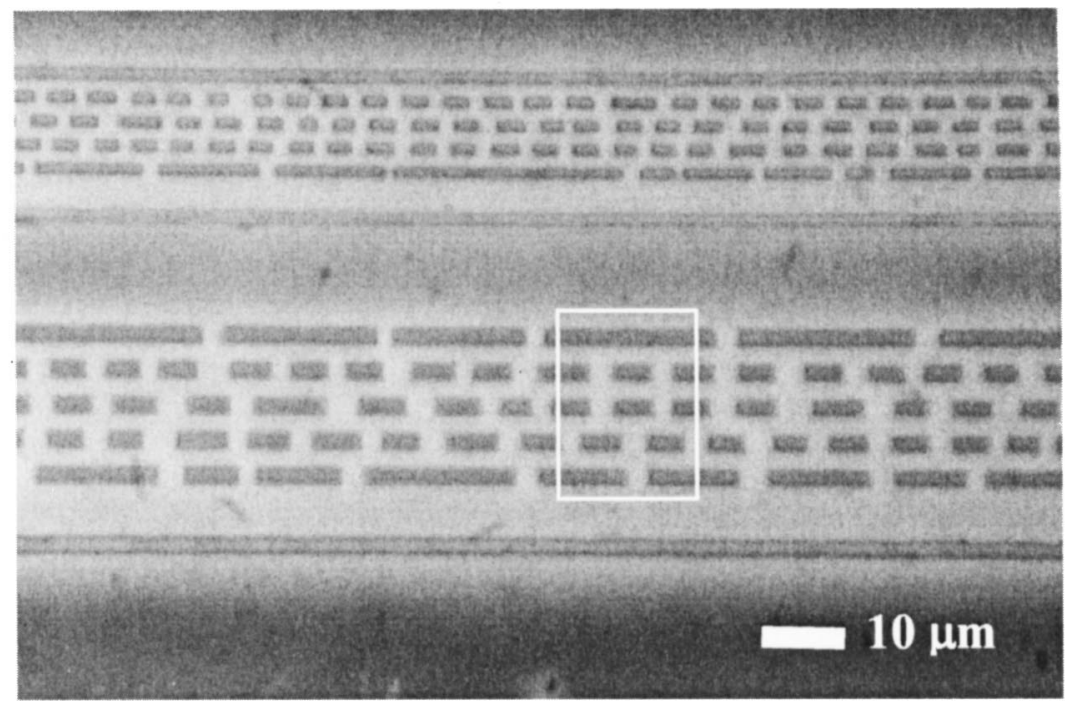

(b)
FIG. 3. (a) Three-dimensional atomic force microscopy (AFM) image of transient morphology of SBS films for $3 \mu \mathrm{m}$ line-and-space pattern [boxed square part in (b)]. Initial film thickness is about $67 \mathrm{~nm}$ and the sample was annealed at $120^{\circ} \mathrm{C}$ for $24 \mathrm{~h}$. Note that some holes are initially generated on the polymer surface and others propagate laterally, contacting one of the mold walls. (b) Optical image of 2 $\mu \mathrm{m}$ (upper group) and $3 \mu \mathrm{m}$ (lower group) line-and-space pattern of the sample in (a). time decreases drastically to $1 \sim 3 \mathrm{~h}$. It is interesting to observe that the outer lines are more stable compared to the inner lines (Fig. 3). The reason the outer lines are more stable has to do with the mass transport associated with dewetting. There is an ample space outside the outer lines, as shown in Fig. 3(b), compared with the inner lines. Therefore, polymer is continuously supplied into the outer lines from the ample space, while the inner lines can draw only from the space between lines during the block formation, which delays the onset of the instability. Mobility also plays a role for the delayed onset of the instability of the outer strips in that the outer strips of SBS are more stable than those of PS. The mobility of SBS, for which the glass transition temperature is $-36{ }^{\circ} \mathrm{C}$, is much larger than that of PS, for which the temperature is $101^{\circ} \mathrm{C}$. Given sufficient time, however, the outer lines of SBS are also separated into regular blocks.

One notable finding in our experiment is that the wavelength of the instability is on the order of $\mu \mathrm{m}$. Capillary wave model predicts that the fastest growing mode in the film is given by

$$
q=a / h^{2},
$$




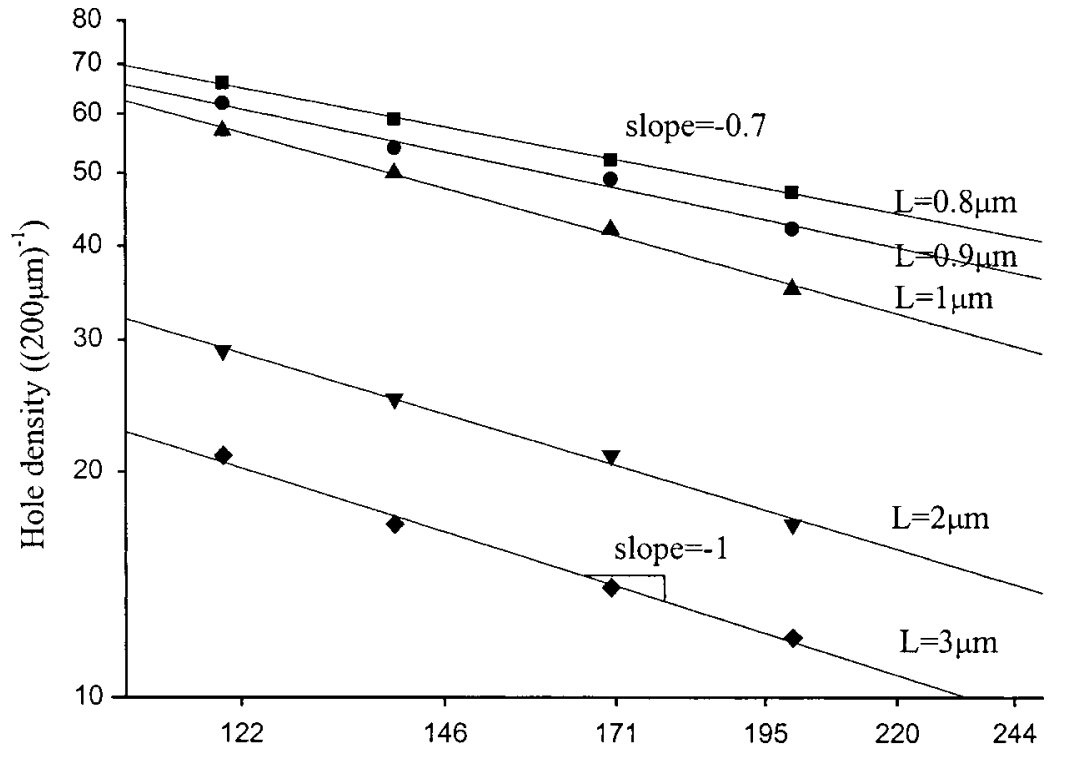

Film thickness in the channel (nm)
FIG. 4. Double logarithmic plots of the hole density of SBS films $\left(36 \mathrm{~h}\right.$ at $\left.120^{\circ} \mathrm{C}\right)$ over a strip length of 200 $\mu \mathrm{m}$ as a function of the film thickness in the channel for given channel width, $L$. For convenience, we assume that the film thickness in the channel is about two times the initial film thickness. The maximum deviation from the average shown in the figure for all data points is less than $\pm 10 \%$ where $a=\left(A_{\text {eff }} / 4 \pi \gamma\right)^{1 / 2}, A_{\text {eff }}$ is the effective Hamaker constant for the van der Waals interaction of the film with the surrounding media, $\gamma$ is the surface tension of the polymer, and $h$ is the film thickness in the channel. The effective Hamaker constant in our experimental geometry should be expressed in terms of three Hamaker constants that are responsible for three interfaces (polymer-air, polymer-substrate, and polymer-PDMS wall). However, its value may range between $10^{-20}$ and $10^{-19}(\mathrm{~J})$ regardless of the individual $\mathrm{Ha}-$ maker constants. ${ }^{12}$ Therefore, the conventional capillary wave model gives a wavelength on the order of several hundred $\mu \mathrm{m}^{1}$ for the conditions used in our experiment. If we adjust the effective Hamaker constant to fit the observed value, it takes a value higher than $10^{-17}(\mathrm{~J})$, which is not realistic. Furthermore, the theory predicts that the hole density in a given strip length should be correlated with $h^{-2}$. To test the dependency, we plot in Fig. 4 the hole density in 200 $\mu \mathrm{m}$ strip length as a function of film thickness in the channel assuming that the thickness in the channel is about two times the initial film thickness. For convenience, we only fit the SBS samples using a simple power law and the resulting straight lines are shown in the figure. As seen in the figure, the power index ranges between -0.7 to -1 , which deviates severely from -2 , which is the slope predicted from the capillary wave model. The power index increases from -1 to -0.7 as the strip width decreases. This fact reveals that the conventional capillary wave model cannot explain our observations. Although not shown, PS films show similar behavior as that of SBS in Fig. 4.

One possible origin that is responsible for this anomaly is the viscoelastic properties of the polymer films. Wang et al. ${ }^{8}$ reported that the power index is not 2 but rather ranges from 0.8 to 1 for high molecular weight polyvinyl-pyridine (PVP) and polystyrene (PS) $\left(M_{w}=9 \times 10^{4}\right)$ thin films. They attributed this anomalous behavior to solid-like or viscoelastic properties of the polymers, as PVP and PS have high molecular weights and strongly react with $\mathrm{SiO}_{2}$ and $\mathrm{Si}$ substrates, respectively. As the molecular weight of the polymer used in our experiment is sufficiently high $\left(>10^{5}\right)$ for both SBS and PS, it would show a more solidlike behavior such that elastic effects may come into play.

Although elastic contribution may be involved in this anomalous behavior, we cannot completely rule out the possibility that the system is dominated by the intermolecular forces. Therefore, it would be valuable to compare the Hamaker constant of our system with that of Ref. 8. For PS, the Hamaker constant in Ref. 8 ranges from $2 \times 10^{-19}$ to 7 $\times 10^{-17}(\mathrm{~J})$ assuming $\gamma$ is $40 \mathrm{~mJ} / \mathrm{m}^{2},{ }^{13}$ which is about two orders of magnitude larger than the generally accepted value $\left[10^{-20}\right.$ and $\left.10^{-19}(\mathrm{~J})\right]$. In our experiment, the value ranges from $6 \times 10^{-18}$ to $3 \times 10^{-17}(\mathrm{~J})$, which is about one order of magnitude larger than that in Ref. 8. In view of the anomalous Hamaker constant and the severe deviation from the capillary wave model, a new model would be needed to explain the behavior reported here.

In summary, we have shown that laterally confined polymer film dewets from the substrate anisotropically along the strip direction. The dewetting results in the formation of distinctly observable, periodically separated blocks of polymer film. The polymer film becomes unstable above its glass transition temperature and the polymer dewets from the substrate by nucleation of holes. The holes initiated on the polymer surface grow, resulting in the separated blocks. Our data show that the hole density of the laterally confined polymer film cannot be determined by the conventional capillary wave model. We believe that the viscoelastic properties of the polymer may be responsible for the anomalous behavior. The simple experimental method proposed in this paper would be quite useful for the studies of polymer films with anisotropic geometry.

\footnotetext{
${ }^{1}$ F. Brochard-Wyart and J. Daillant, Can. J. Phys. 68, 1084 (1990).

${ }^{2}$ L. Leger and J. F. Joanny, Rep. Prog. Phys. 55, 431 (1992).

${ }^{3}$ G. Reiter, Phys. Rev. Lett. 68, 75 (1992).

${ }^{4}$ A. Milchev and K. Binder, J. Chem. Phys. 106, 1978 (1997).

${ }^{5}$ R. Xie, A. Karim, J. F. Douglas, C. C. Han, and R. A. Weiss, Phys. Rev.
} 
Lett. 81, 1251 (1998).

${ }^{6}$ S. Herminghaus, K. Jacobs, K. Mecke, J. Bischof, A. Fery, M. Ibn-Elhaj, and S. Schlagowski, Science 282, 916 (1998).

7 A. Sharma and R. Khanna, Phys. Rev. Lett. 81, 3463 (1998).

${ }^{8}$ J. Wang, M. Tolan, O. H. Seeck, S. K. Sinha, O. Bahr, M. H. Rafailovich, and J. Sokolov, Phys. Rev. Lett. 83, 564 (1999).

${ }^{9}$ A. M. Higgins and R. A. L. Jones, Nature (London) 404, 476 (2000); M. Gleiche, L. F. Chi, and H. Fuchs, ibid. 403, 173 (2000).
${ }^{10}$ Y. Xia, J. A. Rogers, K. E. Paul, and G. M. Whitesides, Chem. Rev. 99, 1823 (1999)

${ }^{11}$ M. O. David, G. Reiter, T. Sitthai, and J. Schultz, Langmuir 14, 5667 (1998).

12 J. Isaellachivili, Intramolecular Surfaces Forces, 2nd ed. (Academic, New York, 1992).

${ }^{13}$ Polymer Handbook, 3rd ed., edited by J. Brandrup and E. H. Immergut (Wiley, New York, 1989). 